\title{
Communication \\ Performance Comparison of Solid Lead Ion Electrodes with Different Carbon-Based Nanomaterials as Electron-Ion Exchangers
}

\author{
Lei Zhang * $\mathbb{D}$, Zhengying Wei $\mathbb{D}$, Pengcheng Liu, Haoran Wei and Denglong Ma \\ State Key Lab for Manufacturing System Engineering, Xi'an Jiaotong University, Xi'an 710049, China; \\ zywei@mail.xjtu.edu.cn (Z.W.); jerryz2017@stu.xjtu.edu.cn (P.L.); weihaoran@stu.xjtu.edu.cn (H.W.); \\ denglong.ma@xjtu.edu.cn (D.M.) \\ * Correspondence: z1870127@stu.xjtu.edu.cn
}

check for updates

Citation: Zhang, L.; Wei, Z.; Liu, P.; Wei, H.; Ma, D. Performance Comparison of Solid Lead Ion Electrodes with Different

Carbon-Based Nanomaterials as Electron-Ion Exchangers. Sensors 2021, 21, 1663. https://doi.org/ $10.3390 / \mathrm{s} 21051663$

Academic Editors:

Stela-Maria Pruneanu and

Miguel Hernaez

Received: 16 January 2021

Accepted: 22 February 2021

Published: 28 February 2021

Publisher's Note: MDPI stays neutral with regard to jurisdictional claims in published maps and institutional affiliations.

Copyright: (C) 2021 by the authors. Licensee MDPI, Basel, Switzerland. This article is an open access article distributed under the terms and conditions of the Creative Commons Attribution (CC BY) license (https:/ / creativecommons.org/licenses/by/ $4.0 /)$.

\begin{abstract}
Carbon-based nanomaterials with carboxylation or chemical modification are widely used as electron-ion exchangers of solid electrodes. For reducing the complexity and dangerousness of the intermediate layer preparation, different original carbon-based nanomaterials are dispersed in deionized water. They are applied in the fabrication of $\mathrm{Pb}^{2+}$-selective electrodes. Because the contact angle of graphene reached $132.5^{\circ}$, the $\mathrm{Pb}^{2+}$-selective electrode of graphene used as an electron-ion exchanger showed excellent performance with a low detection limit of $3.4 \times 10^{-8} \mathrm{M}$ and a fast average response time of $42.6 \mathrm{~s}$. The Nernstian response slope could reach $26.8 \mathrm{mV} / \mathrm{decade}$, and the lifetime lasted for a month. Therefore, graphene suspension without any treatment can be used as the intermediate layer of solid-state electrodes, providing a reference for the preparation of other ion-selective electrodes.
\end{abstract}

Keywords: $\mathrm{Pb}^{2+}$ selective electrode; graphene; multi-walled carbon nanotube; fullerene

\section{Introduction}

Heavy metals such as lead increasingly aggravate the pollution of ecosystems and the poisoning of organisms [1]. Therefore, it is necessary to monitor the lead content [2]. There are many kinds of techniques for detection and the typical analytical methods include atomic absorption spectrometry (AAS) [3], ultraviolet-visible spectrophotometer [4], high performance liquid chromatography (HPLC) [5] and X-ray fluorescence (XRFS) [6], which exhibit disadvantages such as complex operation, poor portability and expensive instruments. The ion-selective electrodes, especially the all-solid-state ion-selective electrodes (ASS-ISEs), are widely applied in environmental monitoring [7-10] owing to portability, low cost and simple operation [11].

The coated wire electrodes (CWEs), in which a thin polymeric film comprising electroactive ionophore is dip-coated directed on the metal wire [12], are the initial formation of ASS-ISEs. Although the preparation of CWEs is simple, the potentials drift chaotically because of the "blocking" membrane/wire interfaces (Figure 1) and the sensitivity to small accidental charges [13]. The water may penetrate through the sensitive membrane, then a thin water layer is formed on the wire surface. The instability of electrode potentials is caused by the unstable metal ion concentration in the aqueous layer. Therefore, a great deal of research has been focused on the use of an electron-ion exchanger to improve the "blocking" interfaces and electrode performance. Due to high conductivity $[14,15]$, conductive polymers (CPs) are employed as intermediate layers in solid-state ISEs [16]. For instance, the commercially available CPs named polyaniline (PANI) was drop-casted on acetate transparency sheets [17] and another applicable CPs called poly(3,4-ethylenedioxythiophene) (PEDOT) were electrochemically deposited onto magnesium microwires [18]. After drying, the cocktails of ion-selective membrane were coated on the CPs layer. The introduction 
of CPs as an electron-ion exchanger improved the potential stability. However, the formation of a thin water film between the CPs and ion-sensitive membrane resulted in chemical hysteresis [19]. Moreover, the oxidation reaction with dissolved oxygen limited the performance of the CPs-based electrode.

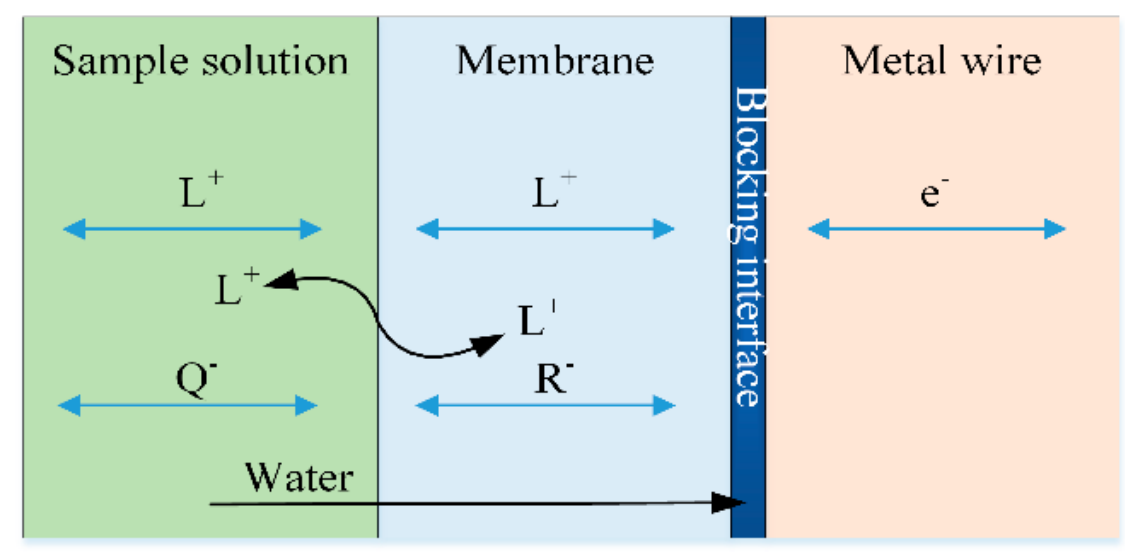

Figure 1. Charge transportation and blocking interface in coated wire electrodes (CWE). $\mathrm{L}^{+}$: cation to be measured; $\mathrm{Q}^{-}$: anion in sample solution, $\mathrm{R}^{-}$: anion in membrane; $\mathrm{e}^{-}$: electron.

Due to the good hydrophobicity and conductivity, carbon-based nanomaterials are widely exploited as electron-ion exchangers instead of CPs. Graphene (GR) (Figure S1a) is a two-dimensional crystal composed of closely packed carbon atoms. It has high strength, good toughness, light weight, high light transmittance, good conductivity and large specific surface area. The main tube of carbon nanotubes (CNTs) (Figure S1b) is formed by curling a part of GR sheets. Fullerene $\left(\mathrm{C}_{60}\right)$ (Figure S1c) is obtained by bending a part of GR into a football shape. Therefore, three carbon-based nanomaterials have been extensively studied and applied to sensors. Potentiometric sensors based on multi-walled carbon nanotube (MWCNT) as inner transducing layer exhibited good resistance to potential drift and the formation of water films [20-22]. $\mathrm{C}_{60}$, an efficient charge-transfer mediator, was used as solid contact layer for ASS-ISEs. The characteristics of the electrode showed that the ion-to-electron transduction and the potential stability were promoted [23,24]. Amr M. Mahmoud fabricated an eco-friendly solid-contact selective electrode with GR as an electron-ion exchanger for the determination of flavoxate hydrochloride; the prepared electrode showed little potential drift and good stability in comparison with the electrode without the graphene layer [25]. Surface groups were produced in the carbon-based nanomaterials by purification, carboxylation and chemical modification [22,25-28]. The carboxylated and modified nanomaterials were suitable as substrates for reacting with specific ions, but the structural damage would reduce their mechanical properties. At the same time, the complexity and dangerousness of material preparation increased. In order to solve these problems, GR, MWCNT and $\mathrm{C}_{60}$ selected were dispersed in the deionized water (DI water), respectively. The suspension was drop-cast on substrate electrode, followed by natural drying to form the electron-ion exchanger. The optimal solid $\mathrm{Pb}^{2+}$-ISE was obtained by comparing the properties of three electrodes with different carbon-based nanomaterials as the intermediate layer.

\section{Experimental}

\subsection{Reagents and Materials}

Glassy carbon (GC) electrodes were purchased from Xuzhou Zhenghao Electronics Co., Ltd. (Xuzhou, China). Reagents including o-nitrophenyl octyl ether (o-NPOE), poly(vinyl chloride) (PVC), anhydrous ethanol and lead nitrate $\left(\mathrm{Pb}\left(\mathrm{NO}_{3}\right)_{2}\right)$ standard solution were obtained from Shanghai Aladdin Industrial Corporation (Shanghai, China). Lead ionophore IV (Figure S2), tetrahydrofuran (THF) and acetone were purchased from Tianjin Kemiou Chemical Reagent Co., Ltd. (Tianjin, China). Potassium chloride ( $\mathrm{KCl}$, 
$>99.5 \%$ ), sodium tetrakis[3,5-bis(trifluoromethyl)phenyl]borate (NaTFPB) and potassium ferricyanide $\left(\mathrm{K}_{3}\left[\mathrm{Fe}(\mathrm{CN})_{6}\right]\right)$ of analytical grade were acquired from Tianjin Tianli Chemical Reagent Co. (Tianjin, China). GR. MWCNT and $\mathrm{C}_{60}$ were provided by Nanjing Ji Cang Nano Technology Co., Ltd. (Nanjing, China), Beijing Tsinghua-Nafine Nano-Powder Commercialization Engineering Center and Nanjing Xianfeng Nano Material Technology Co., Ltd. (Nanjing, China), respectively. The corresponding solutions were prepared in DI water (resistance 18.25 M $\Omega \cdot \mathrm{cm}$, ULUPURE, Chengdu, China).

\subsection{ISEs Fabrication}

The glassy carbon (GC) electrodes with a diameter of $3 \mathrm{~mm}$ were polished in aqueous dispersions of alumina powder $(0.5,0.3$ and $0.05 \mu \mathrm{m}$, respectively). Then, the GC electrodes were ultrasonically cleaned in DI water and anhydrous ethanol for 3 min separately and dried under nitrogen. Finally, the cyclic voltammetry characteristics of GC electrodes were measured in $\mathrm{K}_{3}\left[\mathrm{Fe}(\mathrm{CN})_{6}\right]$ solution to obtain a qualified substrate electrode pretreated (in Section 2.3). Carbon-based nanomaterial (GR, MWCNT and $\mathrm{C}_{60}$, respectively) was dispersed by ultrasonic vibration in DI water for $12 \mathrm{~h}$ to form a $1 \mathrm{mg} / \mathrm{mL}$ uniform suspension. The electron-ion exchanger was acquired by drop casting $50 \mu \mathrm{L}$ carbon-based nanomaterial dispersion on the qualified GC electrodes, followed by drying at room temperature (yielding GC/GR, GC/MWCNT and GC/C 60 electrodes). The $\mathrm{Pb}^{2+}$ ion-selective membrane cocktail (total mass $250 \mathrm{mg}$ ) was prepared by dissolving lead ionophore IV $(1.4 \mathrm{wt} \%)$, NaTFPB ( $0.6 \mathrm{wt} \%)$, plasticizer (63 wt \%, o-NPOE) and PVC (35 wt $\%)$ in $2 \mathrm{~mL}$ THF. Subsequently, $20 \mu \mathrm{L}$ cocktail was drop-cast onto the GC/GR, GC/MWCNT and GC/C60 electrodes evenly and the solvent was evaporated thoroughly at room temperature (producing GC/GR/ $/ \mathrm{Pb}^{2+}$-ISE, GC/MWCNT/ $/ \mathrm{Pb}^{2+}$-ISE and GC/C $60 / \mathrm{Pb}^{2+}$-ISE).

For the measurements, the fabricated electrodes were conditioned in $10^{-3} \mathrm{M} \mathrm{Pb}\left(\mathrm{NO}_{3}\right)_{2}$ solution for at least $12 \mathrm{~h}$ and then in $10^{-9} \mathrm{M} \mathrm{Pb}\left(\mathrm{NO}_{3}\right)_{2}$ solution for more than $24 \mathrm{~h}$.

\subsection{Apparatus and Measurements}

The morphologies of GR, MWCNT and $\mathrm{C}_{60}$ suspension were tested using field emission scanning electron microscope (FESEM) (SU8010, Hitachi, Tokyo, Japan).

Contact angle (CA) measurements were determined with an optical contact angle measuring instrument (DSA100, KRUSS, Hamburg, German). Approximately $2 \mu \mathrm{L}$ DI water was placed on the electron-ion exchanger formed by different nanomaterials. CA values were calculated automatically by the equipment matching software.

The cyclic voltammograms and potential responses were measured with an electrochemical workstation (PARSTAT3000, Ametek, Berwyn, PA, USA) in a three-electrode system: working electrode-GC/GR/Pb ${ }^{2+}$-ISE (GC/MWCNT/ $\mathrm{Pb}^{2+}$-ISE or GC/C $60 / \mathrm{Pb}^{2+}$ ISE); reference electrode $-\mathrm{Hg} / \mathrm{Hg}_{2} \mathrm{Cl}_{2}$ / saturated $\mathrm{KCl}$ electrode (SCE); counter electrodeplatinum wire electrode. The pretreated GC electrodes were tested by cyclic voltammetry in the potential range from $-0.1 \mathrm{~V}$ to $0.6 \mathrm{~V}$ at a constant scan rate of $50 \mathrm{mV} / \mathrm{s}$ in $1 \mathrm{mM}$ $\mathrm{K}_{3}\left[\mathrm{Fe}(\mathrm{CN})_{6}\right]$ solution. The potential difference $(<80 \mathrm{mV})$ between oxidation peak and reduction peak is a criterion for judging the qualification of the pretreatment. The stability of $\mathrm{Pb}^{2+}$-ISEs were measured by cyclic voltammetry (scan potential $-0.5 \mathrm{~V}-0.5 \mathrm{~V}$, scan rate $50 \mathrm{mV} / \mathrm{s}$, scan circle 20) in a $0.1 \mathrm{M} \mathrm{KCl}$ solution. The calibration curves of electromotive force (EMF) were obtained in the gradient $\mathrm{Pb}\left(\mathrm{NO}_{3}\right)_{2}$ solutions from low to high concentrations $\left(10^{-11}\right.$ to $\left.10^{-3} \mathrm{M}\right)$.

\section{Results and Discussions}

\subsection{Micrograph of ElectronIon Exchanger}

Figure 2 shows the SEM im-ages of different carbon-based nanomaterials used as the electron-ion exchanger. At $1 \mathrm{~K}$ magnification, $\mathrm{C}_{60}$ are only distributed in a small area, and the size difference is obvious (Figure $2 \mathrm{~g}$ ) for the reason that the dispersibility of $\mathrm{C}_{60}$ in anhydrous ethanol is very bad. At the same magnification, GR is more evenly distributed and has a more consistent size (Figure 2a) than MWCNT (Figure 2d). As shown 
in Figure $2 b, c, e, f, h, i$, flake GR is stacked and distributed in the conducting layer uniformly, MWCNT is aggregated seriously and entwined and $\mathrm{C}_{60}$ is distributed in clumps. Therefore, suspension of GR is more suitable for fabricating the electron-ion exchanger.

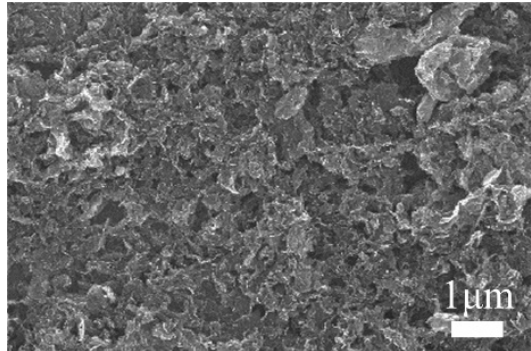

(a)

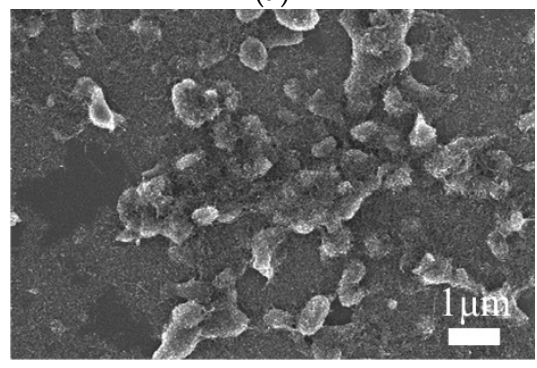

(d)

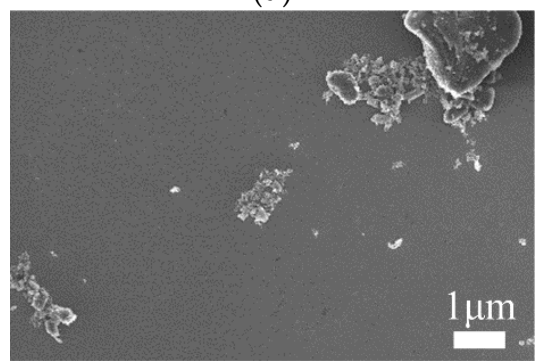

(g)

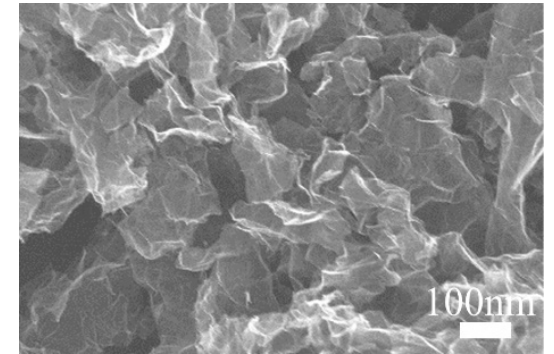

(b)

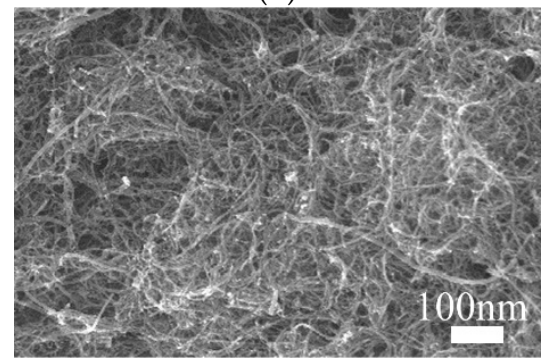

(e)

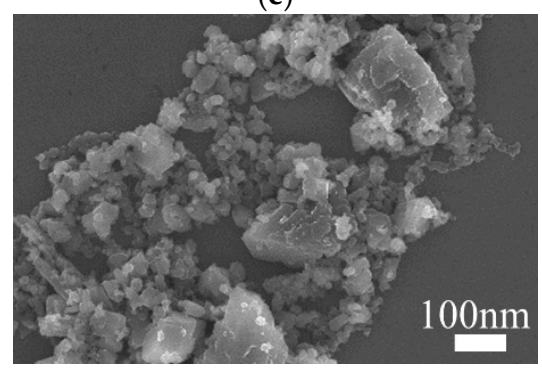

(h)

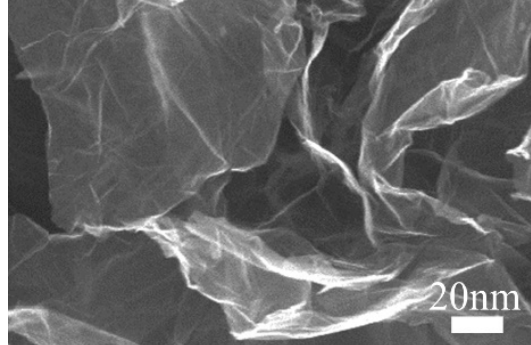

(c)

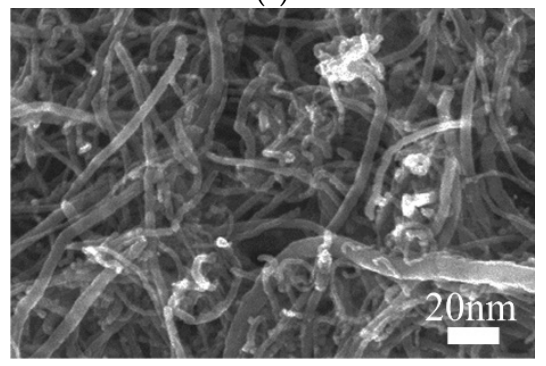

(f)

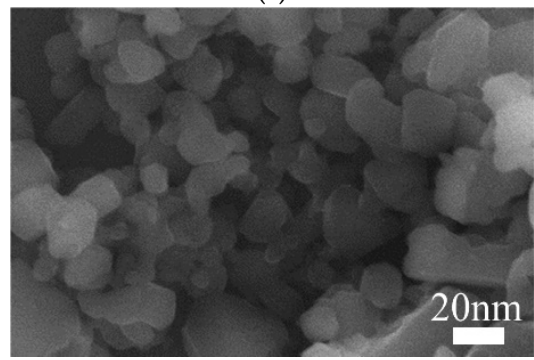

(i)

Figure 2. SEM images of the carbon-based nanomaterials at different magnifications. (a) Graphene (GR) $\times 1 \mathrm{~K}$. (b) GR $\times 10 \mathrm{~K}$. (c) GR $\times 50 \mathrm{~K}$. (d) Multi-walled carbon nanotube $(\mathrm{MWCNT}) \times 1 \mathrm{~K} .(\mathbf{e}) \mathrm{MWCNT} \times 10 \mathrm{~K} .(\mathbf{f}) \mathrm{MWCNT} \times 50 \mathrm{~K} .(\mathrm{g}) \mathrm{C}_{60} \times 1 \mathrm{~K}$. (h) $\mathrm{C}_{60} \times 10 \mathrm{~K}$. (i) $\mathrm{C}_{60} \times 50 \mathrm{~K}$.

\subsection{CA of Carbon-Based Nanomaterials}

Wettability [29] is the capacity of the liquid to spread on a solid surface. CA is an important method to characterize the wettability. The solid-liquid CA (Figure S3) is the angle between the boundary tangent of a droplet and a solid surface. An intermediate layer (electron-ion exchanger) introduced between the sensitive membrane and the substrate electrode plays a significant role in inhibiting the formation of the water layer. Hence, the $\mathrm{CA}$ of electron-ion exchanger directly affects the shielding effect for the water layer. The $90^{\circ} \mathrm{CA}$ is the critical point between hydrophilicity and hydrophobicity. If the CA is more than $90^{\circ}$, the hydrophobicity is better.

As indicated in Figure 3, the CA of GC is $64.9 \pm 0.4^{\circ}$ and the water layer is formed easily because of the high hydrophilicity. The $\mathrm{CA}$ of the $\mathrm{Pb}^{2+}$ ion-sensitive membrane $\left(\mathrm{Pb}^{2+}-\mathrm{ISM}\right)$ is close to $90^{\circ}$. Without a hydrophobic electron-ion exchanger, the performance of the electrode will be affected. GR, MWCNT and $\mathrm{C}_{60}$ electron-ion exchanger were prepared, respectively, then their $\mathrm{CAs}$ were measured. Figure 3 shows that the $\mathrm{CA}$ of the $\mathrm{C}_{60}$ electron-ion exchanger is larger than that of GC. However, the CA is $76.3 \pm 0.3^{\circ}$ (less than $90^{\circ}$ ) and the $\mathrm{C}_{60}$ intermediate layer is manifested as hydrophilicity. It is consistent with the results of micrograph. The CAs of GR and MWCNT are more than $90^{\circ}$ and their electron-ion exchangers show hydrophobicity. The CA in MWCNT electron-ion exchanger 
is $109.8 \pm 0.4^{\circ}$, so its hydrophobicity is weak. The CA of GR is $132.5 \pm 0.4^{\circ}$ and the hydrophobicity is optimal.

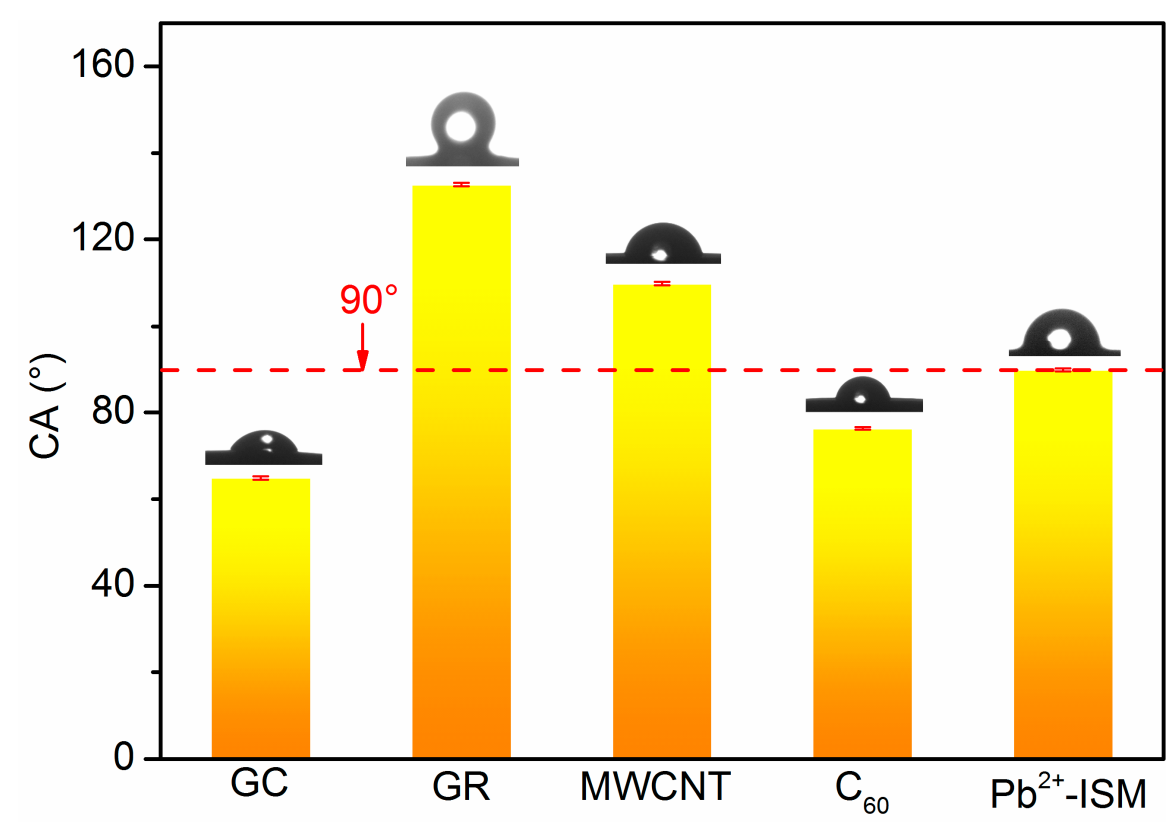

Figure 3. Contact angle (CA) of different carbon-based nanomaterials intermediate layer.

\subsection{Potentiometric Performance}

The potentiometric responses of $\mathrm{Pb}^{2+}$-ISE containing different carbon-based nanomaterials electron-ion exchangers were investigated in the range of $10^{-11}$ to $10^{-3} \mathrm{M}$. As shown in Figure 4, all ISEs are insensitive to the change of low $\mathrm{Pb}\left(\mathrm{NO}_{3}\right)_{2}$ solution concentrations (below $10^{-8} \mathrm{M}$ ). However, the evident EMF step-up change is observed with the increase in $\mathrm{Pb}^{2+}$ concentration at high concentrations (e.g., above $10^{-7} \mathrm{M}$ ). Figure 5 demonstrates that the proposed electrodes show a Nernstian response over a wide range of $10^{-7}$ to $10^{-3} \mathrm{M}$. The low detection limit (LDL) was calculated as the intersection of the two liner lines (the inset in Figure 5). The Nernstian slope and LDL of four electrodes were recorded in Table 1. It can be seen that the characteristics of the electrodes with carbon-based nanomaterials electron-ion exchangers are superior to those of the electrode without an electron-ion exchanger. The GC/GR/ $/ \mathrm{Pb}^{2+}$-ISE shows a Nernstian response of $26.8 \pm 0.3 \mathrm{mV} /$ decade over a wide range of $10^{-7}$ to $10^{-3} \mathrm{M}$. The LDL calculated is $3.4 \times 10^{-8} \mathrm{~mol} / \mathrm{L}$. Response times of all ISEs in the range of Nernstian response are compared in Figure 6. The GR electron-ion exchanger produced less response time. Compared with the response time of $\mathrm{GC} / \mathrm{Pb}^{2+}-\mathrm{ISE}$, the average response time of GC/GR/ $/ \mathrm{Pb}^{2+}-\mathrm{ISE}$ was reduced by $57 \%$. The value was $42.6 \mathrm{~s}$. These results proved the hydrophobicity was GR $>\mathrm{MWCNT}>\mathrm{C}_{60}$, which was consistent with the tests of CA. The potentiometric performance of GC/GR/Pb $/{ }^{2+}-\mathrm{ISE}$ was the best. The performance of GC/GR/ $\mathrm{Pb}^{2+}$-ISE with different thickness of $\mathrm{Pb}^{2+}-\mathrm{ISM}$ is shown in Table S1. 


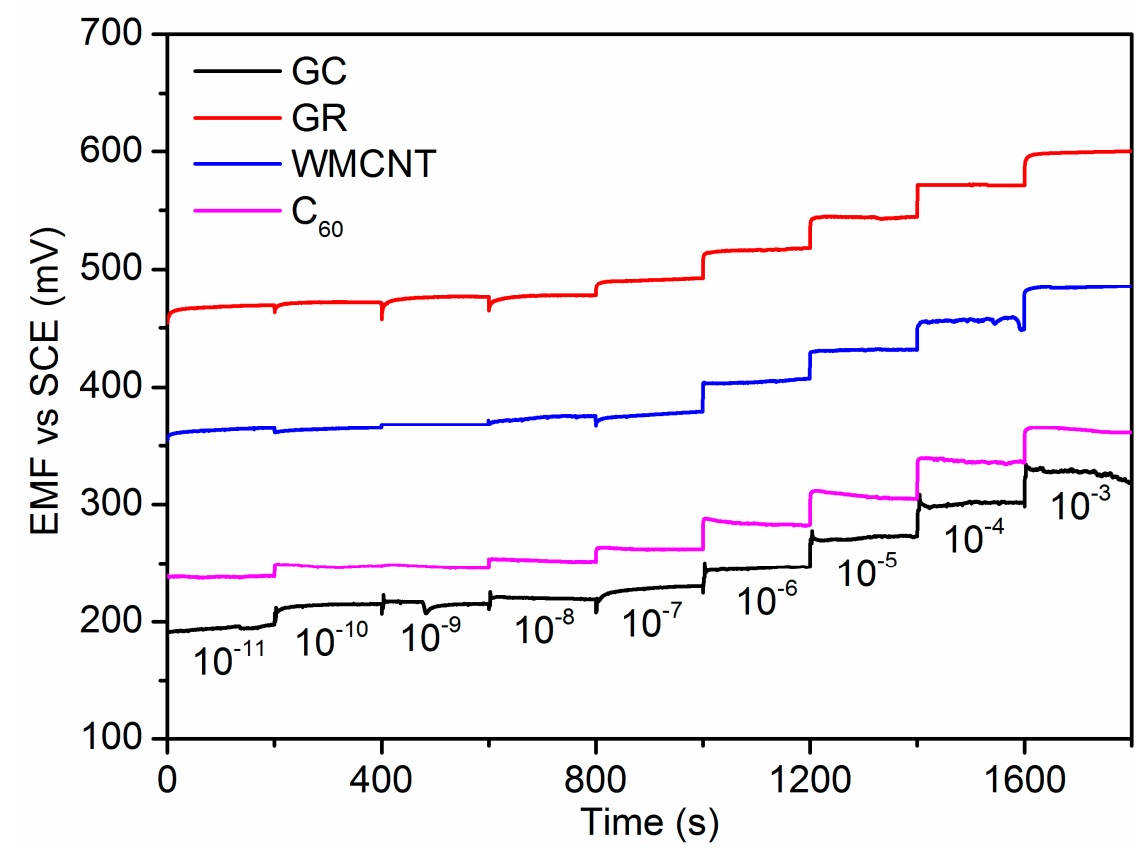

Figure 4. Potentiometric response of the different $\mathrm{Pb}^{2+}$-ion-selective electrodes (ISEs) with lead concentrations from $10^{-11}$ to $10^{-3} \mathrm{M}$.

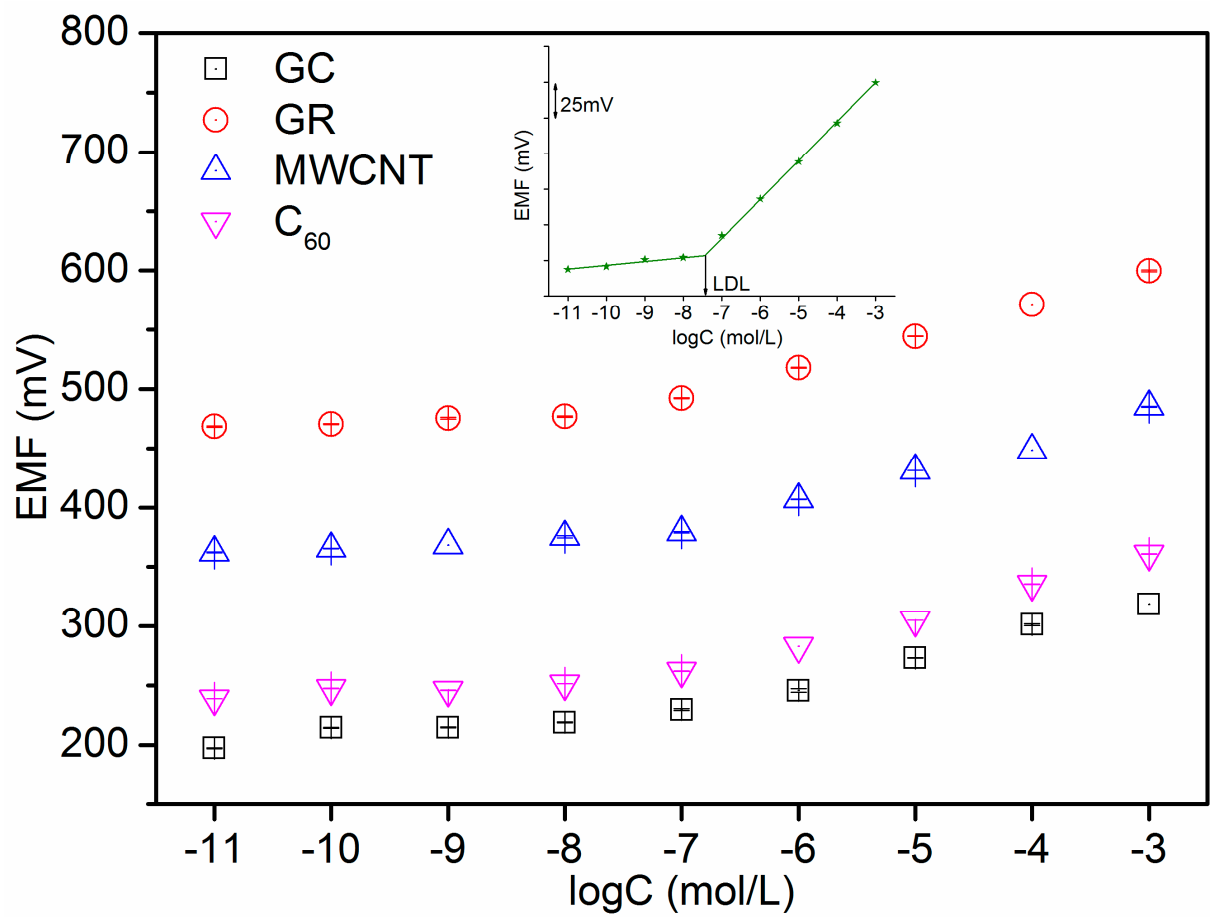

Figure 5. Potentiometric calibration curve of different $\mathrm{Pb}^{2+}$-ISEs in lead concentration range from $10^{-11}$ to $10^{-3} \mathrm{M}$.

Table 1. Comparison of different $\mathrm{Pb}^{2+}$-ISEs characteristics.

\begin{tabular}{ccc}
\hline ISE & Slope (mV/Decade) & LDL (mol/L) \\
\hline $\mathrm{GC} / \mathrm{Pb}^{2+}$-ISE & $23.2 \pm 1.3$ & $1.1 \times 10^{-7}$ \\
$\mathrm{GC} / \mathrm{GR} / \mathrm{Pb}^{2+}$-ISE & $26.8 \pm 0.3$ & $3.4 \times 10^{-8}$ \\
$\mathrm{GC} / \mathrm{MWCNT} / \mathrm{Pb}^{2+}$-ISE & $25.4 \pm 1.5$ & $8.7 \times 10^{-8}$ \\
$\mathrm{GC} / \mathrm{C}_{60} / \mathrm{Pb}^{2+}$-ISE & $25.0 \pm 1.1$ & $6.4 \times 10^{-8}$ \\
\hline
\end{tabular}




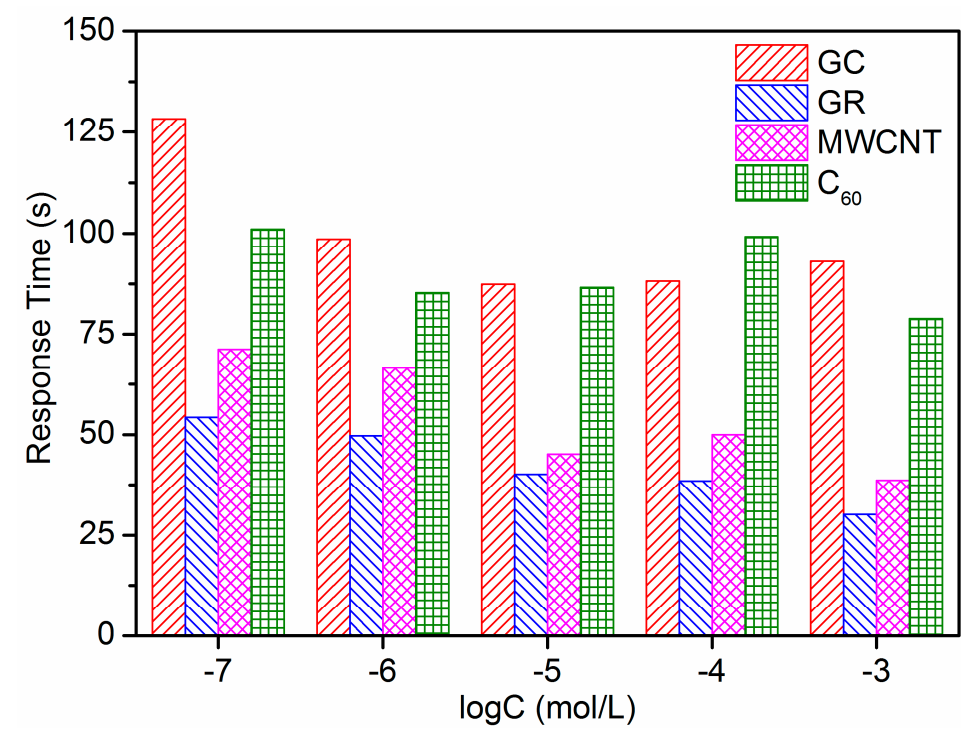

Figure 6. Potentiometric response time of different $\mathrm{Pb}^{2+}-$ ISEs in the range of Nernstian response.

\subsection{Stability and Conductivity of ISEs}

In the practical measurements, the stability of ISE has great influence on the accuracy due to the drift of potential. Multi-circle cyclic voltammetry scan was performed in a $0.1 \mathrm{M} \mathrm{KCl}$ solution. The 20-circle scan curves of each electrode have only slight offset as shown in Figure 7; hence, the prepared electrodes display good stability. In Section 2.2, the carbon-based nanomaterials dispersion is easy to prepare. However, there are some defects in the dispersion uniformity, which are also consistent with the morphologies of SEM. In the $10^{-5} \mathrm{~mol} / \mathrm{L}$ lead solution, GC/GR/ $/ \mathrm{Pb}^{2+}$-ISE and GC/MWCNT/ $\mathrm{Pb}^{2+}$-ISE were tested for $3000 \mathrm{~s}$, respectively. The potentials remain stable as shown in Figure S4. The currents of GC/GR/ $\mathrm{Pb}^{2+}$-ISE and GC/MWCNT/ $\mathrm{Pb}^{2+}$-ISE are higher than those obtained at $\mathrm{GC} / \mathrm{C}_{60} / \mathrm{Pb}^{2+}$-ISE and GC/Pb ${ }^{2+}$-ISE because GR and MWCNT have a large electrontransfer ability and high specific surface area. Furthermore, the lead ion electrode with GR as the intermediate layer has the best conductivity.

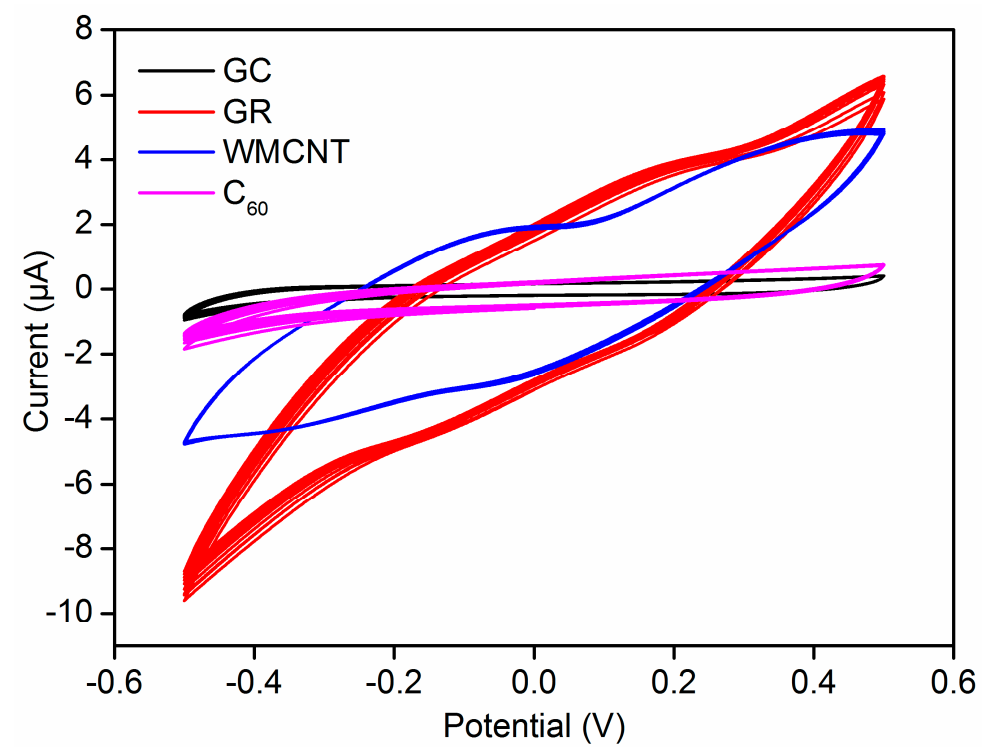

Figure 7. Multi-circle cyclic voltammetry curves of different $\mathrm{Pb}^{2+}-$ ISEs. 


\subsection{Lifetime of ISEs}

The long-period potential responses of the fabricated electrodes were tested to obtain the Nernstian slope in the range of $10^{-7} \mathrm{~m}$ to $10^{-3} \mathrm{M} \mathrm{Pb}^{2+}$ solution. As shown in Figure 8 , the response slopes of $\mathrm{GC} / \mathrm{C}_{60} / \mathrm{Pb}^{2+}$-ISE and GC/ $/ \mathrm{Pb}^{2+}$-ISE decrease by half rapidly after two weeks. No obvious loss of response slopes is observed within a week for GC/GR/ $/ \mathrm{Pb}^{2+}$-ISE and GC/MWCNT/ $\mathrm{Pb}^{2+}$-ISE. After 28 days, the response slopes of GC/GR/ $\mathrm{Pb}^{2+}$-ISE and GC/MWCNT/ $\mathrm{Pb}^{2+}$-ISE reduce by $10.3 \%$ and $25.6 \%$, respectively. However, the electrode with GR as intermediate layer still presents a Nernstian response slope of more than $24 \mathrm{mV} /$ decade. The robust and reliable GC/GR/Pb ${ }^{2+}$-ISE is promising for applications in many fields such as drinking water and industrial wastewater. When the prepared electrodes are not in use, they should be dipped in the conditioning solution of $10^{-9} \mathrm{M} \mathrm{Pb}\left(\mathrm{NO}_{3}\right)_{2}$.

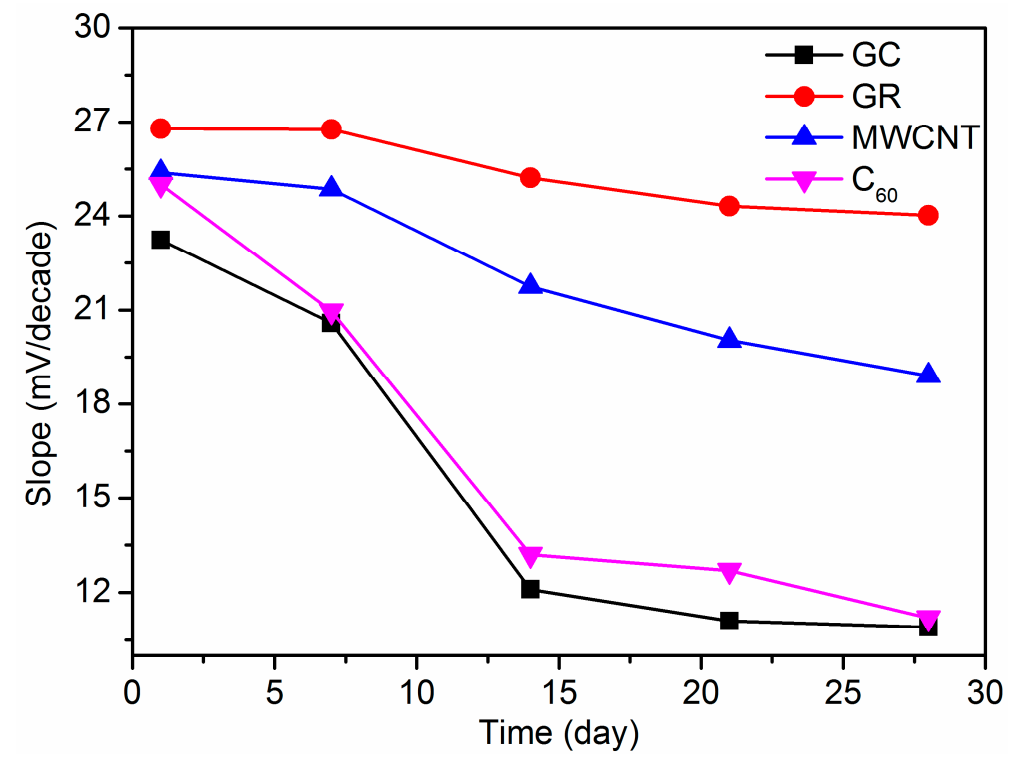

Figure 8. Long-time slope of different $\mathrm{Pb}^{2+}$-ISEs.

\subsection{Comprehensive Property and Follow-up Studies}

The properties of the four electrodes manufactured in this paper are compared with those in the literatures. As shown in Table 2, five indexes including linear range, slope, LDL, response time and lifetime represent the performances of electrodes. The $\mathrm{Pb}^{2+}$ electrode with GR as electron-ion exchanger has better comprehensive performance and is suitable for testing a $\mathrm{Pb}^{2+}$ solution of low concentration.

Table 2. Comparison of comprehensive performance for different $\mathrm{Pb}^{2+}$ electrodes.

\begin{tabular}{|c|c|c|c|c|c|c|}
\hline No. & $\begin{array}{l}\text { Linear Range } \\
(\mathrm{mol} / \mathrm{L})\end{array}$ & $\begin{array}{c}\text { Slope } \\
\text { (mV/decade) }\end{array}$ & $\begin{array}{c}\text { LDL } \\
(\mathrm{mol} / \mathrm{L})\end{array}$ & $\begin{array}{c}\text { Response Time } \\
\text { (s) }\end{array}$ & $\begin{array}{l}\text { Lifetime } \\
\text { (day) }\end{array}$ & Note \\
\hline 1 & $10^{-7} \sim 10^{-3}$ & 23.24 & $1.10 \times 10^{-7}$ & 99.12 & 1 & $\mathrm{GC} / \mathrm{Pb}^{2+}-\mathrm{ISE}$ \\
\hline 2 & $10^{-7} \sim 10^{-3}$ & 26.79 & $3.44 \times 10^{-8}$ & 42.58 & 28 & $\mathrm{GC} / \mathrm{GR} / \mathrm{Pb}^{2+}$-ISE \\
\hline 3 & $10^{-7} \sim 10^{-3}$ & 25.39 & $8.65 \times 10^{-8}$ & 54.34 & 7 & $\mathrm{GC} / \mathrm{MWCNT} / \mathrm{Pb}^{2+}$-ISE \\
\hline 4 & $10^{-7} \sim 10^{-3}$ & 25.01 & $6.39 \times 10^{-8}$ & 90.22 & 1 & $\mathrm{GC} / \mathrm{C}_{60} / \mathrm{Pb}^{2+}$-ISE \\
\hline 5 & $10^{-5} \sim 10^{-1}$ & 26 & - & 60 & 14 & [30] \\
\hline
\end{tabular}

In addition, the slope, response time and lifetime of GC/GR/ $\mathrm{Pb}^{2+}$-ISE can be improved further for extensive application. 


\section{Conclusions}

This study demonstrated that different carbon-based nanomaterials can be applied in the fabrication of $\mathrm{Pb}^{2+}$-selective electrodes. Especially, the $\mathrm{Pb}^{2+}$-selective electrode of graphene used as an electron-ion exchanger showed excellent performance with a low detection limit of $3.4 \times 10^{-8} \mathrm{M}$ and a fast average response time of $42.6 \mathrm{~s}$. The Nernstian response slope could reach $26.8 \mathrm{mV} /$ decade. The addition of a graphene intermediate layer in the $\mathrm{Pb}^{2+}$-selective electrode enhanced the hydrophobicity which was testified by contact angle measurement. Moreover, the GC/GR/ $/ \mathrm{Pb}^{2+}$-ISE displayed good potential stability and conductivity. Furthermore, the lifetime of the electrode lasted for a month. When the graphene suspension was fabricated for the electron-ion exchanger of $\mathrm{Pb}^{2+}$-selective electrode, the potential performance and lifetime could be improved. Therefore, graphene can be used as the intermediate layer of solid-state electrodes, providing a reference for the preparation of other ion-selective electrodes.

Supplementary Materials: The following are available online at https:/ / www.mdpi.com/1424-8 220/21/5/1663/s1, Figure S1: Schematic of carbon-based nanomaterial structure, Figure S2: The structure of the lead ionophore IV, 4-tert-butylcalix[4]arene-tetrakis( $\mathrm{N}, \mathrm{N}$-dimethylthioacetamide), Figure S3: Schematic of a sessile-drop contact angle system, Table S1: Comparison of GC/GR/ $\mathrm{Pb}^{2+}$ ISEs with different volume of $\mathrm{Pb}^{2+}$-ISM characteristics, Figure S4: EMF of GC/GR/ $\mathrm{Pb}^{2+}-\mathrm{ISE}$ and $\mathrm{GC} / \mathrm{MWCNT} / \mathrm{Pb}^{2+}$-ISE in $10^{-5} \mathrm{~mol} / \mathrm{L}$ lead solution.

Author Contributions: Methodology, L.Z. and P.L.; validation, H.W. and D.M.; investigation, L.Z. and P.L.; data curation, L.Z. and P.L.; writing-original draft preparation, L.Z.; writing-review and editing, L.Z., P.L. and H.W.; supervision, Z.W. and D.M.; funding acquisition, Z.W. All authors have read and agreed to the published version of the manuscript.

Funding: This research was funded by the National Key Research and Development Plan of China, grant number 2016YFC0400202.

Institutional Review Board Statement: Not applicable.

Informed Consent Statement: Not applicable.

Data Availability Statement: All data included in this study are available upon request by contact with the corresponding author.

Conflicts of Interest: The authors declare no conflict of interest.

\section{References}

1. Chon, H.-T.; Ahn, J.S.; Jung, M.C. Heavy Metal Contamination in the Vicinity of Some Base-Metal Mines in Korea; a Review. Geosyst. Eng. 1998, 1, 74-83. [CrossRef]

2. Korn, M.D.G.A.; De Andrade, J.B.; De Jesus, D.S.; Lemos, V.A.; Bandeira, M.L.; Dos Santos, W.N.; Bezerra, M.A.; Amorim, F.A.; Souza, A.S.; Ferreira, S.L. Separation and preconcentration procedures for the determination of lead using spectrometric techniques: A review. Talanta 2006, 69, 16-24. [CrossRef] [PubMed]

3. Assubaie, F.N. Assessment of the levels of some heavy metals in water in Alahsa Oasis farms, Saudi Arabia, with analysis by atomic absorption spectrophotometry. Arab. J. Chem. 2015, 8, 240-245. [CrossRef]

4. Guo, Y.; Liu, X.; Han, Y.; Bian, X.; Zhang, Q. Effective Enrichment and Simultaneous Quantitative Analysis of Trace Heavy Metal Ions Mixture in Aqueous Samples by the Combination of Radial Electric Focusing Solid Phase Extraction, UV-Vis Spectrophotometric Determination and Partial Least Squares Regression. Waterairsoil Pollut. 2017, 228, 317. [CrossRef]

5. Ali, I.; Aboul-Enein, H.Y. Speciation of arsenic and chromium metal ions by reversed phase high performance liquid chromatography. Chemosphere 2002, 48, 275-278. [CrossRef]

6. Zarazua, G.; Ávila-Pérez, P.; Tejeda, S.; Barcelo-Quintal, I.; Martínez, T. Analysis of total and dissolved heavy metals in surface water of a Mexican polluted river by total reflection X-ray fluorescence spectrometry. Spectrochim. Acta Part B At. Spectrosc. 2006, 61, 1180-1184. [CrossRef]

7. Zuliani, C.; Diamond, D. Opportunities and challenges of using ion-selective electrodes in environmental monitoring and wearable sensors. Electrochim. Acta 2012, 84, 29-34. [CrossRef]

8. Eriksen, R.S.; Mackey, D.J.; Van Dam, R.; Nowak, B. Copper speciation and toxicity in Macquarie Harbour, Tasmania: An investigation using a copper ion selective electrode. Mar. Chem. 2001, 74, 99-113. [CrossRef]

9. Crespo, G.A. Recent Advances in Ion-selective membrane electrodes for in situ environmental water analysis. Electrochim. Acta 2017, 245, 1023-1034. [CrossRef] 
10. Hanrahan, G.; Patil, D.G.; Wang, J. Electrochemical sensors for environmental monitoring: Design, development and applications. J. Environ. Monit. 2004, 6, 657-664. [CrossRef]

11. Bobacka, J.; Ivaska, A.; Lewenstam, A. Potentiometric Ion Sensors. Chem. Rev. 2008, 108, 329-351. [CrossRef] [PubMed]

12. Cattrall, R.W.; Tribuzio, S.; Freiser, H. Potassium ion responsive coated wire electrode based on valinomycin. Anal. Chem. 1974, 46, 2223-2224. [CrossRef] [PubMed]

13. Janata, J. Principles of Chemical Sensors; Springer Science \& Business Media: Atlanta, GA, USA, 2010; pp. 119-122.

14. Kaur, G.; Adhikari, R.; Cass, P.; Bown, M.; Gunatillake, P.A. Electrically conductive polymers and composites for biomedical applications. RSC Adv. 2015, 5, 37553-37567. [CrossRef]

15. Li, W.; Zhang, Q.; Zheng, G.; Seh, Z.W.; Yao, H.; Cui, Y. Understanding the Role of Different Conductive Polymers in Improving the Nanostructured Sulfur Cathode Performance. Nano Lett. 2013, 13, 5534-5540. [CrossRef]

16. Bobacka, J. Conducting Polymer-Based Solid-State Ion-Selective Electrodes. Electroanalysis 2006, 18, 7-18. [CrossRef]

17. Michalska, A.; Wojciechowski, M.; Jędral, W.; Bulska, E.; Maksymiuk, K. Silver and lead all-plastic sensors-polyaniline vs. poly (3, 4-ethyledioxythiophene) solid contact. J. Solid State Electrochem. 2009, 13, 99-106. [CrossRef]

18. Zhang, C.; Driver, N.; Tian, Q.; Jiang, W.; Liu, H. Electrochemical deposition of conductive polymers onto magnesium microwires for neural electrode applications. J. Biomed. Mater. Res. Part A 2018, 106, 1887-1895. [CrossRef]

19. Fibbioli, M.; Morf, W.E.; Badertscher, M.; de Rooij, N.F.; Pretsch, E. Potential drifts of solid-contacted ion-selective electrodes due to zero-current ion fluxes through the sensor membrane. Electroanal. Int. J. Devoted Fundam. Pract. Asp. Electroanal. 2000, 12, 1286-1292.

20. Yuan, D.; Anthis, A.H.C.; Afshar, M.G.; Pankratova, N.; Cuartero, M.; Crespo, G.A.; Bakker, E. All-Solid-State Potentiometric Sensors with a Multiwalled Carbon Nanotube Inner Transducing Layer for Anion Detection in Environmental Samples. Anal. Chem. 2015, 87, 8640-8645. [CrossRef]

21. Mousavi, Z.; Teter, A.; Lewenstam, A.; Maj-Żurawska, M.; Ivaska, A.; Bobacka, J. Comparison of Multi-walled Carbon Nanotubes and Poly(3-octylthiophene) as Ion-to-Electron Transducers in All-Solid-State Potassium Ion-Selective Electrodes. Electroanalysis 2011, 23, 1352-1358. [CrossRef]

22. Parra, E.J.; Crespo, G.A.; Riu, J.; Ruiz, A.; Rius, F.X.; Arnó, E.J.P. Ion-selective electrodes using multi-walled carbon nanotubes as ion-to-electron transducers for the detection of perchlorate. Analyst 2009, 134, 1905-1910. [CrossRef]

23. Fouskaki, M.; Chaniotakis, N. Fullerene-based electrochemical buffer layer for ion-selective electrodes. Analyst 2008, 133, 1072-1075. [CrossRef]

24. Li, J.; Yin, T.; Qin, W. An all-solid-state polymeric membrane Pb2+-selective electrode with bimodal pore C60 as solid contact. Anal. Chim. Acta 2015, 876, 49-54. [CrossRef] [PubMed]

25. Mahmoud, A.M.; Ragab, M.T.; Ramadan, N.K.; El-Ragehy, N.A.; El-Zeany, B.A. Design of Solid-contact Ion-selective Electrode with Graphene Transducer Layer for the Determination of Flavoxate Hydrochloride in Dosage Form and in Spiked Human Plasma. Electroanalysis 2020, 32, 2803-2811. [CrossRef]

26. Ghaedi, M.; Jaberi, S.Y.S.; Hajati, S.; Montazerozohori, M.; Asfaram, A.; Mirtamizdoust, B.; Zare, M. CuO nanoparticles intermixed with chemically modified multiwalled carbon nanotubes as a novel electrode for $\mathrm{Cu} 2+$ ion determination. IEEE Sen. J. 2014, 15, 2882-2890.

27. Barathi, P.; Senthil Kumar, A. Electrochemical Conversion of Unreactive Pyrene to Highly Redox-Active 1,2-Quinone Derivatives on a Carbon Nanotube-Modified Gold Electrode Surface and Its Selective Hydrogen Peroxide Sensing. Langmuir 2013, 29, 10617-10623. [CrossRef]

28. Mai, C.-C.; Shih, J.-S. Bifunctional Ion-Selective Electrode Based on C60-Cryptand 22 for Silver and Iodine Ions. J. Chin. Chem. Soc. 2012, 59, 1031-1038. [CrossRef]

29. Darmanin, T.; Guittard, F. Wettability of conducting polymers: From superhydrophilicity to superoleophobicity. Prog. Polym. Sci. 2014, 39, 656-682. [CrossRef]

30. Jaber, A.M.Y.; Moody, G.J.; Thomas, J.D.R. Studies on lead ion-selective electrodes based on polyalkoxylates. Analyst 1988, 113, 1409-1413. [CrossRef] 